\title{
OPTICAL FLOW RECOGNITION FROM THE POWER SPECTRUM OF A SINGLE BLURRED IMAGE
}

\author{
Ioannis M. Rekleitis \\ Center for Intelligent Machines, School of Computer Science, \\ McGill University, 3480 University St., Montreal, Québec, \\ Canada H3A 2A7, e-mail: yiannis@cs.mcgill.ca
}

\begin{abstract}
In this paper a new technique for calculation of the optical flow is presented. When there is motion in the observed scene, an image taken will be motion blurred (to a degree depending on the exposure time). Up to now most of the algorithms for estimating the motion in a scene ignored motion blur and treated it as noise. On the contrary, motion blur is structured information and in certain cases can be used to infer the velocities locally. This new approach uses the information of the motion blur in the frequency domain to extract the orientation and the magnitude of the velocity - optical flow. ${ }^{1}$
\end{abstract}

\section{INTRODUCTION}

One of the fundamental problems in early Computer Vision is the measurement of motion in an image, frequently called optical flow. In most biological visual systems, the analysis of motion is critical. During the last twenty years many algorithms have been proposed to calculate the optical flow. An early approach is that of Horn and Schunck [9], who used a differential approach. Since then many other algorithms have been proposed. These can be divided into different categories according to the way they handle the data used to calculate the optical flow. The most general categories are: differential methods, correspondence methods, and region based matching, in 2D or 3D [1], [15], [3]. The problem of estimating the optical flow has received much attention because of its many different applications. Tasks such as passive scene interpretation, image segmentation [10], surface structure reconstruction, inference of egomotion, and active navigation [8], [12] all use optical flow as input information.

\footnotetext{
${ }^{1}$ Appeared in "IEEE Signal Processing Society, International Conference in Image Processing", Lausanne, Switzerland, Sep. 1996.
}

\section{BACKGROUND}

When the exposure time for the image is large enough, it is inevitable that there will be blur in the image due to relative motion between the camera and the scene. Most of the proposed algorithms up to now either ignore motion blur or treat it as another source of noise. Consequently, most motion estimation algorithms consider optical flow with displacements of only a few pixels per frame, and most of them use more than one frame. This limits the applications to slower motions and fails to seriously address the issue of motion blur; moreover, it works on images that are considered to be taken with infinitely small exposure time, more or less in a "stop and shoot" approach. This can be a limitation in real time applications. It is worth noting that the use of multiple images increases the amount of data needed, thus demanding more memory and taking more time. Also, as most of these algorithms work on a series of images by calculating the displacement of every pixel from image to image, they ignore any information about motion that exists within each single image. As the blur created by motion extends only across the orientation of the motion and for as much as the magnitude, it is clear that information about the velocity vector could be extracted from it. Motion blur is of particular interest in the biological research and many studies about its significance in the perception of the world have been done [2], [7], [4]. Earlier work in the estimation of the motion blur parameters has used different methods such as the bispectrum [5], or the Discrete Cosine Transform [16]; in both cases the orientation of the motion was assumed known. In this work we avoid this rather unrealistic simplifying assumption.

\section{ALGORITHM DESCRIPTION}

In this paper we have developed and evaluated a new approach to the problem of visual motion estimation. The algorithm we have developed is based on interpreting the cue of motion blur to estimate the optical flow 
field in a single image. A key observation is that motion blur introduces a characteristic structure, a ripple (figure 1b), in the Fourier transform, that has the same orientation as the motion and period equivalent to the magnitude of the blur. This can be detected and quantified using a modified form of cepstral analysis. Unlike classical approaches to visual motion analysis that rely upon operators tuned to specific spatial and temporal frequencies at specific orientations, our new approach makes use of all the information that can be gathered from a patch of the image and is thus quite robust.

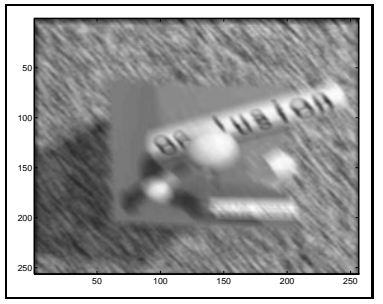

(a)

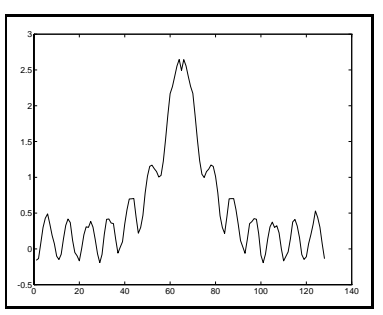

(c)

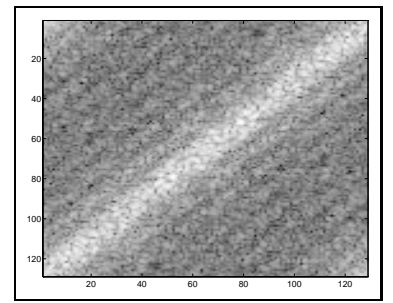

(b)

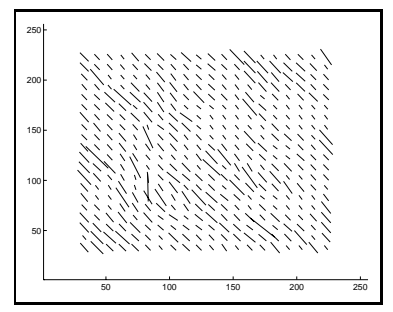

(d)
Figure 1: A natural image artificially blurred (a), its Fourier Spectrum (b), the Fourier Spectrum collapsed (c), and the Optical flow Map (d)

In order to calculate the velocity vector in a position we take an image patch around the position, mask it with a Gaussian filter in order to reduce the ringing effect, and zero pad it. The first step in our motion blur analysis is to compute the logarithm of the magnitude of the Fourier Transform of that image patch. Motion blur leads to a tell-tale ripple, centered at the origin, stretched across the orientation of the velocity vector (figure 1b). This orientation can be reliably determined, even in the presence of noise, by using a second Gaussian derivative filter aligned to the orientation of the motion. In order to do this we have to calculate the response of the Power Spectrum in all orientations and choose the maximum, this is possible in $\mathrm{O}(\mathrm{n})$ as the second derivative of the Gaussian filter belongs to a family called "steerable filters" [6], whose response $R G_{2}^{\theta}$ can be calculate at any angle $\theta$ (as in equation 1 ) based only on the responses of three basis filters (see table 1). The angle at which this filter gives the maximum response is the orientation of the velocity vector.

$$
R G_{2}^{\theta}=k_{a}(\theta) R G_{2 a}+k_{b}(\theta) R G_{2 b}+k_{c}(\theta) R G_{2 c}
$$

\begin{tabular}{|l|l|}
\hline$G_{2 a}=0.921\left(2 x^{2}-1\right) e^{\mu}$ & $k_{a}(\theta)=\cos ^{2}(\theta)$ \\
\hline$G_{2 b}=1.843 x y e^{\mu}$ & $k_{b}(\theta)=-2 \cos (\theta) \sin (\theta)$ \\
\hline$G_{2 c}=0.921\left(2 y^{2}-1\right) e^{\mu}$ & $k_{c}(\theta)=\sin ^{2}(\theta)$ \\
\hline$\mu=-\left(x^{2}+y^{2}\right)$ & \\
\hline
\end{tabular}

Table 1: The three basis filters and their interpolation functions

The two dimensional signal is too noisy to provide robust results for the magnitude of the motion. Therefore, the magnitude of the velocity vector, which is related to the period of the ripple, can then be determined by first collapsing the log spectrum data into an one dimensional signal (see figure 1c). As can be seen by comparing the figures $1 \mathrm{c}$ and $2 \mathrm{a}$ the shape of the one dimensional signal is almost identical to that of the $\operatorname{sinc}$ function $\left(\operatorname{sinc}(x)=\frac{\sin (x)}{x}\right)$. This comes as no surprise as the sinc function is the result of the Fourier Transform on the one dimensional blur. Consequently, in order to extract the period of the ripple a second Fourier transform is performed, thus providing the Cepstrum of the signal [11], in which the magnitude of the velocity is clearly identified by a negative peak (see figure 2b) [13] [14].

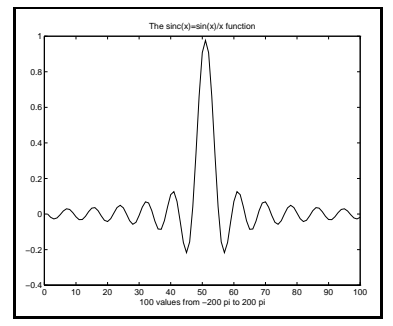

(a)

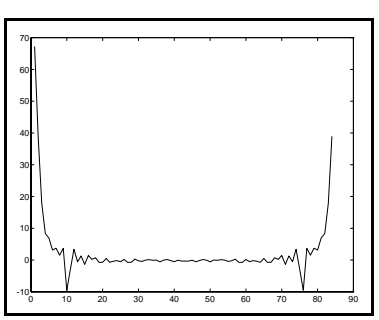

(b)
Figure 2: The sinc function (a), the Cepstrum of the collapsed signal (b)

The computational complexity of this algorithm is bounded by the Fast Fourier Transform operation, 


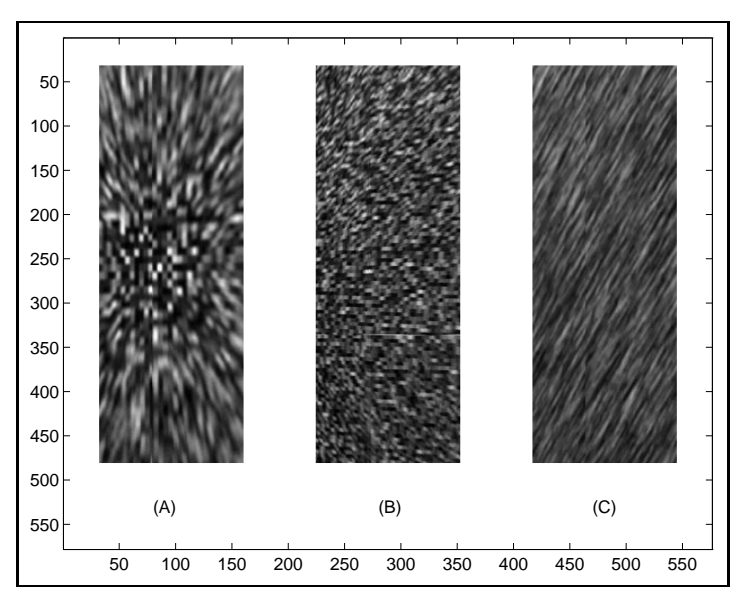

(a)

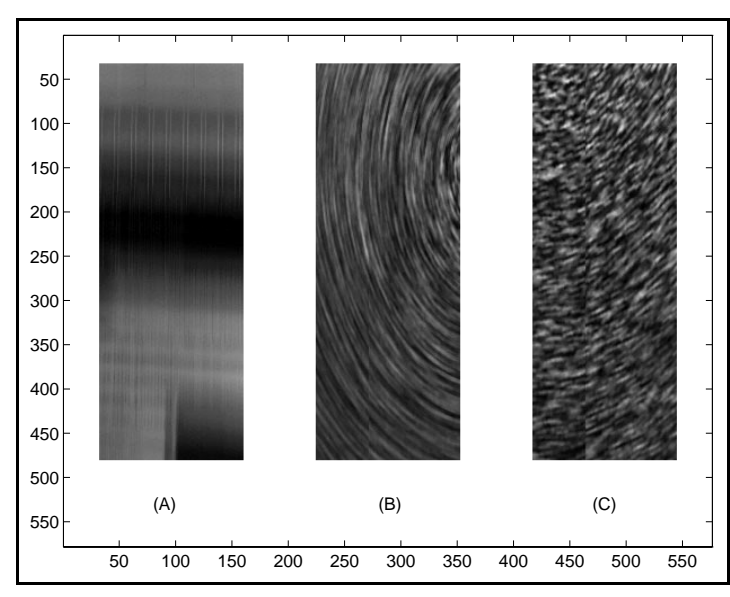

(c)

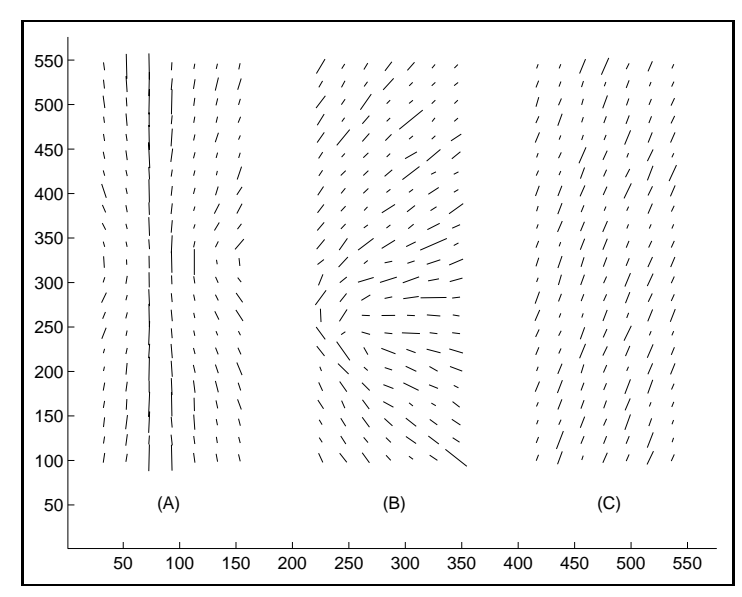

(b)

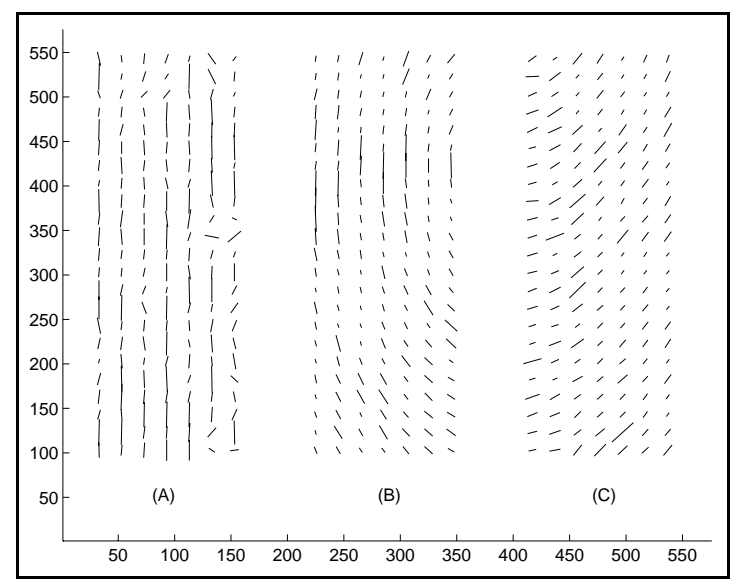

(d)

Figure 3: Real World experiments.

which is $O(n \log n)$, where $n$ is the number of pixels in the image patch. Applying this analysis throughout the image provides an estimation of the complete optical flow field. In figure $1 \mathrm{~d}$ we could see the Optical Flow map of the image in figure $1 \mathrm{a}$, when a window $64 \times 64$ was used over a grid of 10 pixels. A hardware implementation of the FFT could accelerate the process.

\section{RESULTS}

Several experiments have been conducted using stationary images artificially blurred (see fig. 1a, velocity map fig. 1d), and real images taken by a camera with the existence of relative motion between the cam- era and the scene. In the case of synthetic images a digital image was convolved with a kernel simulating the effects of motion blur (for better results the kernel was created by using anti-aliasing line techniques). The orientation was recovered with an average error of $1^{\circ}-3^{\circ}$, and the magnitude to $4-6$ pixels.

In the experiments with real world pictures a different setup was used. The camera was held steady and a board painted with random dots was moved relative to it. In the figure $3 \mathrm{a}$ three instances have been recorded, in $(A)$ and $(B)$ the board is moving away from the camera (dilation) and in $(C)$ it is moving parallel to the imaging plane (translation). The orientation and the magnitude have been calculated quite accurately in the velocity maps in figure $3 \mathrm{~b}$. More precisely, in 
(A) the center of expansion is accurately positioned at the center of the image, while in $(B)$ it can be seen at the left side just bellow the center of the image. In figure $3 \mathrm{c}$ the first picture $(A)$ is taken by translating the camera across a bookshelf, motion that is clearly visible in the velocity map. The second two pictures $(B)$ and $(C)$ have been taken with the board spinning under the camera, motion that is accurately displayed in their velocity maps accordingly (Fig. 3d), while the centers of rotation could be located in both cases. Although, no quantitative error measures could be obtained, as the motion were not controlled, an examination of the the velocity maps proves them qualitatively correct. Moreover, the qualitative observation in conjunction with the numerical results from the synthetic images, shows that the algorithm accurately describes the Optical Flow.

\section{SUMMARY}

In this paper a new approach calculating the optical flow map using motion blur is formulated and evaluated experimentally. An algorithm is presented for computing the optical flow from a single motion-blurred image, using only the information present in the structure imposed on the image by the motion blur. The results acquired are very promising: the orientation of the velocity vector is accurately estimated $\left(1^{\circ}\right.$ to $3^{\circ}$ average error), and the magnitude calculations are satisfied for qualitative estimations. Our algorithm has the advantage of exploiting information in a motion-blurred image that traditional motion analysis methods tended to ignore. It has also the added advantage of providing an optical flow map from a single image, instead of a sequence of images.

\section{REFERENCES}

[1] J. K. Aggarwal and N. Nandhakumar. On the computation of motion from sequences of images-a review. Proceedings of the IEEE, 76(8):917-935, August 1988.

[2] Charles H. Anderson. Blur into focus. Nature, 343:419420, February 1990.

[3] J. L. Barron, D. J. Fleet, and S. S. Beauchemin. Performance of optical flow techniques. Technical Report TR299; RPL-TR-9107, Dept. of Computer Science, University of Western Ontario; Dept. of Computer Science, Queens University, London, Ontario, N6A 5B7; Kingston, Ontario, K7L 3N6, July 1992.

[4] C. Bonnet. Visual motion detection models:features and frequency. Perception, 6:491-500, 1977.

[5] Michael M. Chang, Murat A. Tekalp, and Tanju A. Erdem. Blur identification using the bispectrum. IEEE Transactions on Signal Processing, 39(10):2323-2325, Octomber 1991.
[6] William T. Freeman and Edward H. Adelson. The design and use of steerable filters. IEEE Transactions on Pattern Analysis and Machine Intelligence, 13(9):891906, September 1991.

[7] Thomas L. Harrington and Marcia K. Harrington. Perception of motion using blur pattern information in the moderate and high-velocity domains of vision. Acta Psychologica, 48:227-237, 1981.

[8] Martin Herman and Tsai-Hong Hong. Visual navigation using optical flow. In Proc. NATO Defense Research Group Seminar on Robotics in the Battlefield, pages 1-9. NATO, Paris France, March 1991.

[9] Berthold K. P. Horn and Brian G. Schunck. Determining optical flow. Technical report, Massachusetts Institute of Technology, 1980.

[10] H. A. Mallot, H. H. Bulthoff, J. J. Little, and S. Bohrer. Inverse perspective mapping simplifies optical flow computation and obstacle detection. Biological Cybernetics, 64:177-185, 1991.

[11] William K. Pratt. Digital Image Processing. John Wiley \& Sons, Inc., 1978.

[12] K. Prazdny. Egomotion and relative depth map from optical flow. Biological Cybernetics, 36:87-102, 1980.

[13] Ioannis M. Rekleitis. Visual motion estimation based on

motion blur interpretation. Master's thesis, School of Computer Science, McGill University, Montreal, Quebec, Canada, 1995, http://www.cim.mcgill.ca/ ${ }^{\sim}$ yiannis/Publications/thesis.ps.gz.

[14] Ioannis M. Rekleitis. Steerable filters and cepstral analysis for optical flow calculation from a single blurred image. In Vision Interface, pages 159-166, Toronto, May 1996, http://www.cim.mcgill.ca/ yiannis/Publications/vi96a.ps.gz.

[15] J. F. Vega-Riveros and K. Jabbour. Review of motion analysis techniques. IEE Proceedings, 136(6):397-404, December 1989.

[16] Yasuo Yoshida, Kazuyochi Horiike, and Kazuhiro Fujita. Parameter estimation of uniform image blur using dct. IEICE Trans. Fundamentals, E76(7):1154-1157, July 1993. 